\title{
Quantum Potential Approaches for Nano-scale Device Simulation
}

\author{
HIDEAKI TSUCHIYA, BRIAN WINSTEAD and UMBERTO RAVAIOLI*
}

Beckman Institute, University of Illinois at Urbana-Champaign, Urbana, IL 61801, USA

\begin{abstract}
With the progress of integrated technology, the feature size of experimental electron devices have already been scaled down deeply into the sub $-0.1 \mu \mathrm{m}$ region. For such ultra-small devices, it is increasingly important to take quantum mechanical effects into account for device simulation. In this paper, we present a new approach for quantum modeling, applicable to multi-dimensional ultra-small device simulation. In this work, the quantum effects are represented in terms of quantum mechanically corrected potential in the classical Boltzmann equation. We apply the Monte Carlo method to solve the quantum transport equation, and demonstrate that the quantum effects such as tunneling and quantum confinement effects can be incorporated in the standard Monte Carlo techniques.
\end{abstract}

Keywords: Monte Carlo simulation; Quantum effects; Wigner function

\section{INTRODUCTION}

With the remarkable progress of integrated technology, an oxide thickness of less than $1.5 \mathrm{~nm}$ is needed in MOS devices, where direct tunneling occurs increasing gate leakage current. Another issue is the loss of inversion charge and transconductance due to the quantization of carriers in the channel as well as poly-Si gate depletion. Deep scaling is also expected to bring new effects such as current enhancement due to tunneling through the source/drain barrier. From the device modeling point of view, high-end physical simulations such as particle Monte Carlo solution of the Boltzmann transport equation (BTE) have become more and more important to understand nonequilibrium and hot carrier phenomena and to calibrate models at lower levels in the hierarchy such as drift-diffusion approach [1]. However, the BTE is a fully classical equation though collisions are calculated quantum mechanically. So, the conventional Monte Carlo procedures have been unable to embody the carrier quantum transport effects in a self-contained way. In this paper, we present a new approach for quantum modeling based upon the Wigner transport formalism, where the quantum

*Address for correspondence: 3255 Beckman Institute, University of Illinois, 405 North Mathews Avenue, Urbana, IL 61801, USA. Tel.: + 217-244-5765, Fax: + 217-244-4333, e-mail: ravaioli@uiuc.edu 
effects are represented in terms of quantum mechanically corrected potential in the classical BTE [2]. We apply the Monte Carlo method to solve it, and demonstrate that the quantum effects can be incorporated in the standard Monte Carlo techniques.

\section{QUANTUM-CORRECTED BTE}

Similar to the semi-classical Boltzmann transport theory, the Wigner distribution function is defined in the space and momentum coordinates. The transport equation for the Wigner distribution function is given in the form of a modified BTE as [3]

$$
\begin{aligned}
\frac{\partial f}{\partial t}+\mathbf{v} & \cdot \nabla_{\mathbf{r}} f-\frac{1}{\hbar} \nabla_{\mathbf{r}} U \cdot \nabla_{\mathbf{k}} f \\
& +\sum_{\alpha=1}^{\infty} \frac{(-1)^{\alpha+1}}{\hbar 4^{\alpha}(2 \alpha+1) !}\left(\nabla_{\mathbf{r}} U \cdot \nabla_{\mathbf{k}} f\right)^{2 \alpha+1}=\left(\frac{\partial f}{\partial t}\right)_{C}
\end{aligned}
$$

An essential consideration of the Wigner formalism is the entry of nonlocal quantum effects through the inherently nonlocal driving potential in the fourth term on the left-handside. In the limit of slow spatial variations, the nonlocal terms disappear and Eq. (1) reduces to the BTE. Although the Wigner transport equation (1) provides a general and powerful tool, it is limited to one-dimensional applications for its computational cost. So, we consider the lowest-order quantum correction by taking only the $\alpha=1$ term in Eq. (1). Recently, it was shown that the lowest-order approximation is sufficiently accurate for nonequilibrium quantum transport analysis [2]. Further, when the system is close to equilibrium, a displaced Maxwell-Boltzmann distribution is introduced in the correction term as $f=\exp \{-\beta$ $\left.\left[E_{\mathbf{k}-\overline{\mathbf{k}}}+U(\mathbf{r})-E_{f}\right]\right\}$, where $\beta=1 / k_{B} T, \quad E_{\mathbf{k}-\overline{\mathbf{k}}}=$ $\sum_{i=x, y, z} \hbar^{2}\left(k_{i}-\bar{k}_{i}\right)^{2} /\left(2 m_{i}\right)$, and $\bar{k}_{i}$ is the shifted momentum center of the distribution function. After some manipulations, the quantum-corrected BTE for two-dimensional problems is obtained as follows

$$
\frac{\partial f}{\partial t}+\mathbf{v} \cdot \nabla_{\mathbf{r}} f+\frac{1}{\hbar} \mathbf{F}^{q c} \cdot \nabla_{\mathbf{k}} f=\left(\frac{\partial f}{\partial t}\right)_{C}
$$

where the quantum-corrected driving forces $\mathbf{F}^{q c}=$ $\left(F_{x}^{q c}, F_{y}^{q c}\right)$ are represented by

$$
\begin{aligned}
& \begin{array}{r}
F_{x}^{q c} \\
=-\frac{\partial}{\partial x}\left(U-\frac{1}{24}\left\{\left[\gamma_{x}^{2}\left(k_{x}-\bar{k}_{x}\right)^{2}-3 \gamma_{x}\right] \frac{\partial^{2} U}{\partial x^{2}}\right.\right. \\
\left.\left.+3\left[\gamma_{y}^{2}\left(k_{y}-\bar{k}_{y}\right)^{2}-\gamma_{y}\right] \frac{\partial^{2} U}{\partial y^{2}}\right\}\right)
\end{array} \\
& \begin{aligned}
F_{y}^{q c} \\
=-\frac{\partial}{\partial y}\left(U-\frac{1}{24}\left\{\left[\gamma_{y}^{2}\left(k_{y}-\bar{k}_{y}\right)^{2}-3 \gamma_{y}\right] \frac{\partial^{2} U}{\partial y^{2}}\right.\right. \\
\left.\left.+3\left[\gamma_{x}^{2}\left(k_{x}-\bar{k}_{x}\right)^{2}-\gamma_{x}\right] \frac{\partial^{2} U}{\partial x^{2}}\right\}\right)
\end{aligned}
\end{aligned}
$$

with $\gamma_{i}=\beta \hbar^{2} / m_{i}(i=x, y)$. It should be noted that the driving forces are modified so that the particles evolve under the enforcement by the classical builtin potential $U$ plus the quantum-corrected potentials involved with the second derivatives of the potential.

When the classical potential has a discontinuity, for example at potential barriers, then an intensely peaked function will appear on the quantum forces of Eqs. (3) and (4). These strong forces could lead to a very small time step requirement in Monte Carlo simulation. Thus, for practical device simulation, a smooth quantum potential may be desirable. To this end, we introduce the 
approximate relations, $\partial^{2} U / \partial x^{2} \simeq-k_{B} T \partial^{2}(\ln n) /$ $\partial x^{2}$ and similarly for $y$-derivative, where $n$ is the carrier density. These are derived by integrating the displaced Maxwell-Boltzmann distribution with the momentum. As a result, we obtain the alternative representation for the driving forces as follows

$$
\begin{aligned}
& F_{x}^{q c} \\
& \begin{aligned}
=-\frac{\partial}{\partial x}\left(U+\frac{1}{24 \beta}\right. & \left\{\left[\gamma_{x}^{2}\left(k_{x}-\bar{k}_{x}\right)^{2}-3 \gamma_{x}\right] \frac{\partial^{2} \ln n}{\partial x^{2}}\right. \\
+ & \left.\left.3\left[\gamma_{y}^{2}\left(k_{y}-\bar{k}_{y}\right)^{2}-\gamma_{y}\right] \frac{\partial^{2} \ln n}{\partial y^{2}}\right\}\right),
\end{aligned}
\end{aligned}
$$

$$
\begin{aligned}
& F_{y}^{q c} \\
& \begin{aligned}
=-\frac{\partial}{\partial y}\left(U+\frac{1}{24 \beta}\right. & \left\{\left[\gamma_{y}^{2}\left(k_{y}-\bar{k}_{y}\right)^{2}-3 \gamma_{y}\right] \frac{\partial^{2} \ln n}{\partial y^{2}}\right. \\
+ & \left.\left.3\left[\gamma_{x}^{2}\left(k_{x}-\bar{k}_{x}\right)^{2}-\gamma_{x}\right] \frac{\partial^{2} \ln n}{\partial x^{2}}\right\}\right),
\end{aligned}
\end{aligned}
$$

Since the carrier density would vary continuously even at the abrupt potential discontinuities, the driving forces (5) and (6) are expected to realize smooth quantum forces.

We should point out that the carrier's wavenumbers, $k_{x}$ and $k_{y}$, are included in (3) and (4) or (5) and (6). This means that the carriers are driven by the quantum forces according to their own momentum. In the previously proposed quantum potential [4], the momentum terms were replaced with the thermal energy as $\hbar^{2}\left(k_{x}-\bar{k}_{x}\right)^{2} / 2 m_{x} \simeq \hbar^{2}$ $\left(k_{y}-\bar{k}_{y}\right)^{2} / 2 m_{y} \simeq k_{B} T / 2$, and then the quantum forces were simply represented by

$$
\begin{aligned}
& F_{x}^{q c}=-\frac{\partial}{\partial x}\left(U-\frac{\hbar^{2}}{12 m_{x}} \frac{\partial^{2} \ln n}{\partial x^{2}}\right) \\
& F_{y}^{q c}=-\frac{\partial}{\partial y}\left(U-\frac{\hbar^{2}}{12 m_{y}} \frac{\partial^{2} \ln n}{\partial y^{2}}\right)
\end{aligned}
$$

In this model, the same quantum forces had been assigned for all particles in each position.

Next, we discuss the relevance of our approach to the quantum hydrodynamic models $[5,6]$. For onedimensional case, we represent the analytical carrier distribution in equilibrium by considering the simple quantum-corrected potential of Eq. (7) as $n(x)=N_{c} e^{-\beta\left[U(x)+U_{c}(x)-E_{f}\right]}=n_{c l}(x) e^{-\beta U_{c}(x)}$, where $U_{c}=-\left(\hbar^{2} / 12 m_{x}\right) \partial^{2}(\ln n) / \partial x^{2}$ and $n_{c l}$ indicates the classical carrier density without the quantum correction. This equation implies that the carrier spatial distribution is modulated under the influence of the quantum correction $U_{c}$. Considering this fact, we obtain the following average kinetic energy

$$
w=\frac{1}{2} m_{x} v^{2}+\frac{3}{2} e^{\beta U_{c}} k_{B} T .
$$

The thermal energy term is modified by the quantum factor $e^{\beta U_{c}}$. Here, we have to mention that when the factor $e^{\beta U_{c}}$ is expanded with respect to $U_{c}$ and only the lowest-order component is considered, the energy representation (8) reduces to the one proposed by Zhou and Ferry [5]. Based upon Eq. (8), a new set of quantum moment equations with temperature notation is derived as follows

$$
\begin{gathered}
\frac{\partial n}{\partial t}+\frac{\partial(n v)}{\partial x}=0 \\
\frac{\partial v}{\partial t}+v \frac{\partial v}{\partial x}=-\frac{q E}{m_{x}}-\frac{1}{n m_{x}} \frac{\partial}{\partial x}\left(n k_{B} T e^{\beta U_{c}}\right)-\frac{v}{\tau_{m}} \\
\frac{\partial T}{\partial t}+e^{-\beta U_{c}} v \frac{\partial}{\partial x}\left(T e^{\beta U_{c}}\right) \\
=-\frac{2}{3} T \frac{\partial v}{\partial x}+\frac{m_{x} v^{2}}{3 k_{B} e^{\beta U_{c}}}\left(\frac{2}{\tau_{m}}-\frac{1}{\tau_{w}}\right)-\frac{T-T_{0}}{\tau_{w}}
\end{gathered}
$$

We can also derive a general drift-diffusion current equation. When $v\left(\partial_{v} / \partial_{x}\right) \simeq 0$ is assumed in steady-state, the current equation is obtained from 
Eq. (10) as

$$
J=n \mu q E+q D \frac{\partial}{\partial x}\left(n e^{\beta U_{c}}\right)
$$

It is worth noting that the drift-diffusion equation based upon the density-gradient theory [6] is derived from Eq. (12) by expanding $e^{\beta U_{c}}$ and taking the lowest-order component as

$$
J=n \mu q E+q D \frac{\partial n}{\partial x}-\frac{\hbar^{2} n \mu}{6 m_{x}} \frac{\partial}{\partial x}\left(\frac{\partial^{2} \sqrt{n} / \partial x^{2}}{\sqrt{n}}\right)
$$

Therefore, the energy Eq. (8) is considered to be a general representation for the quantum electron energy.

\section{SIMULATION RESULTS}

We present now some Monte Carlo solutions of the quantum transport equation proposed in the previous section. As a simulation model we consider a barrier width of $2.5 \mathrm{~nm}$ with energy potential height $0.22 \mathrm{eV}$, at room temperature (300K). Figure 1 shows the computed electron distributions in space and energy at the bias voltage of $0.3 \mathrm{~V}$. Figures (a) and (b) correspond to the new quantum model (5)-(6), and the conventional quantum model (7), respectively. The vertical axis denotes the total electron energy including the quantum-corrected potential. It is found that the tunneling effect through the central barrier and the quantum confinement effect in the triangular potential well are simulated in both models. Here, the quantum confinement effect means that the particles are distributed away from the left barrier interface, and no electrons exist under a certain energy due to the quantized subband formation. By comparing the two figures we notice the two major improvements in Figure 1(a). First, when the new model is employed, a flat energy level appears in the

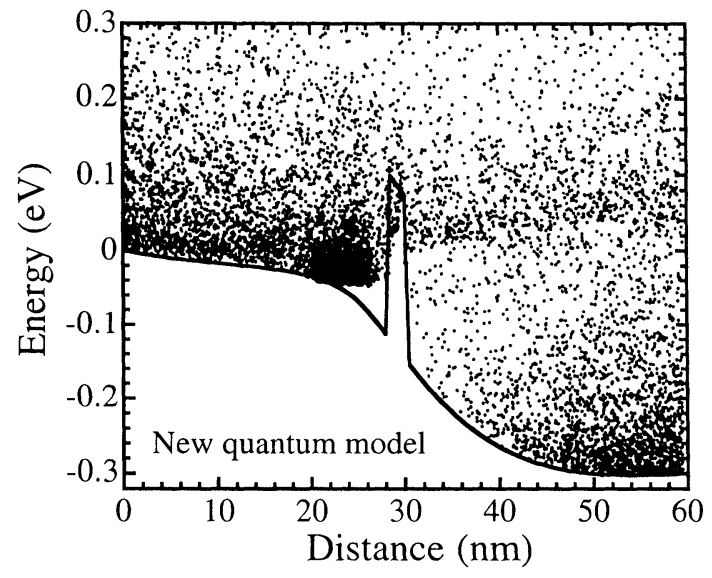

(b)

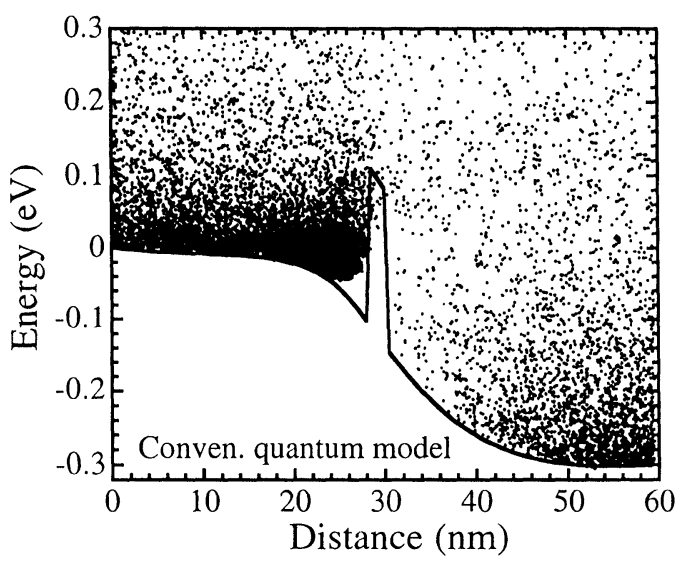

FIGURE 1 Computed particle distributions of $\mathrm{GaAs} / \mathrm{Al}$ GaAs single tunneling barrier at $300 \mathrm{~K}$. (a) corresponds to the new quantum model (5) and (6), and (b) to the conventional quantum model (7). The conduction band profiles are also plotted by the solid lines.

triangular potential well, while the conventional model produces a concave-shaped one. Further, we can find in Figure 1(a) that a larger number of electrons are tunneling through the barrier. Incidentally, in the classical simulation without quantum corrections (not shown here), as one would expect particles exist at the bottom of the potential well and only the thermally excited electrons over the barrier height were found in the barrier region [4]. Consequently, the most important quantum effects, tunneling and quantum confinement, have been incorporated in the 
standard Monte Carlo techniques by considering the quantum-corrected potential in the driving force.

We are also working to extend our method to 2D Monte Carlo simulation for ultra-small MOSFETs. Figure 2 shows a preliminary example, with the computed electron density distributions at the $\mathrm{Si} / \mathrm{SiO}_{2}$ interface of a $25 \mathrm{~nm}$-channel MOSFET. Here, (a) corresponds to the classical Monte Carlo simulation and (b) to the quantum-corrected Monte Carlo simulation with the conventional quantum potential (7). One can see that the classically simulated carriers are positioned very close to the interface in the channel as shown in Figure 2(a). On the other hand, the carriers in the quantum-corrected simulation are traveling with a certain average displacement from the interface, due to the quantum confinement effects as shown in Figure 2(b). These preliminary results indicate that our model should be applicable to practical multi-dimensional simulation. A final remark is that the full band structure of crystals can also be included in our model [4]. Thus, we believe that the proposed approach will be useful to extend the

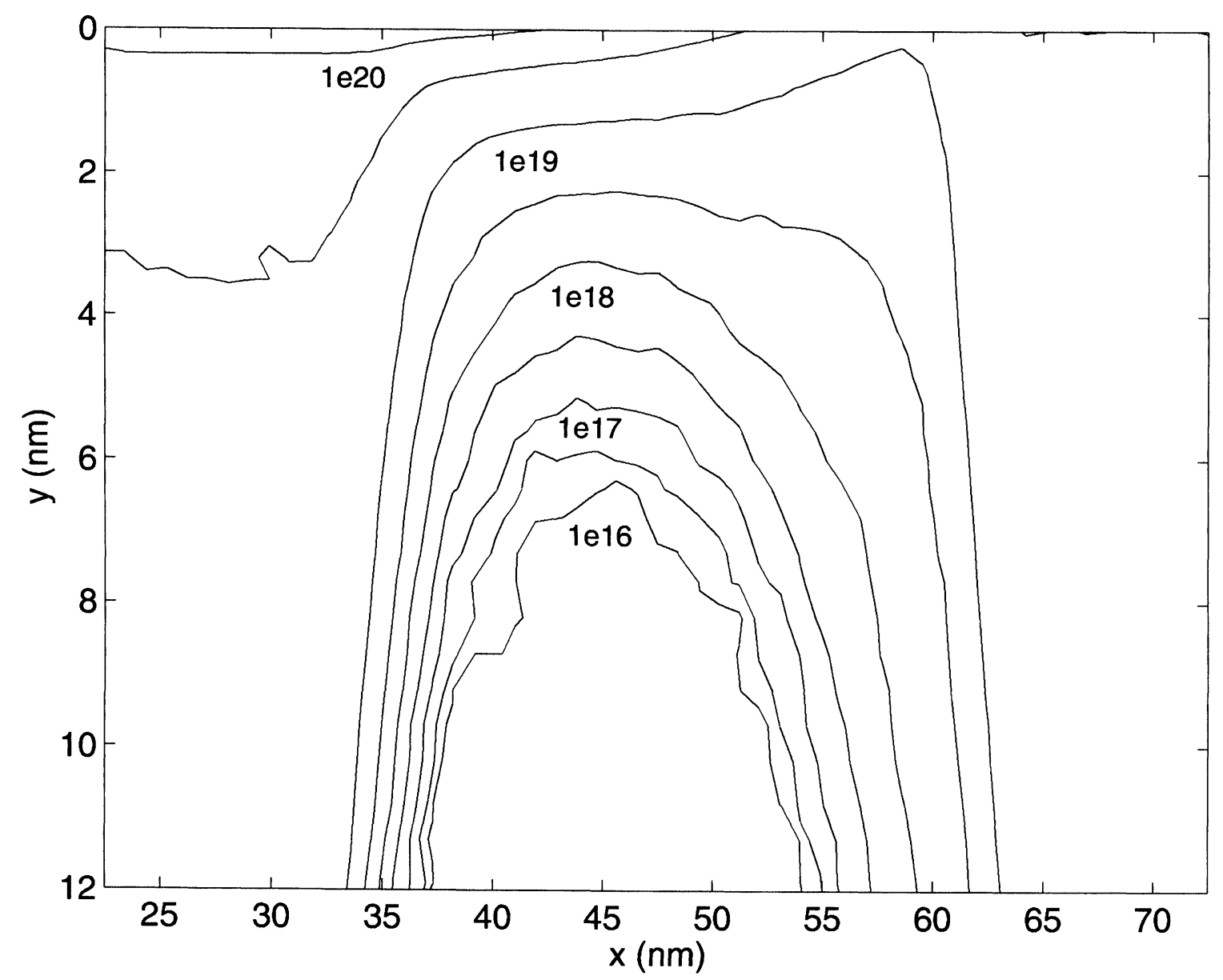

FIGURE 2 Computed electron density distributions at the $\mathrm{Si} / \mathrm{SiO}_{2}$ interface of a $25 \mathrm{~nm}$-channel MOSFET, where (a) corresponds to the semi-classical simulation, (b) to the quantum-corrected Monte Carlo simulation. The $\mathrm{Si} / \mathrm{SiO}_{2}$ interface is located at $y=0$, the oxide thickness is $t_{o x}=1.5 \mathrm{~nm}$, and the applied bias is $V_{G}=1.0 \mathrm{~V}$ and $V_{D}=1.0 \mathrm{~V}$. 


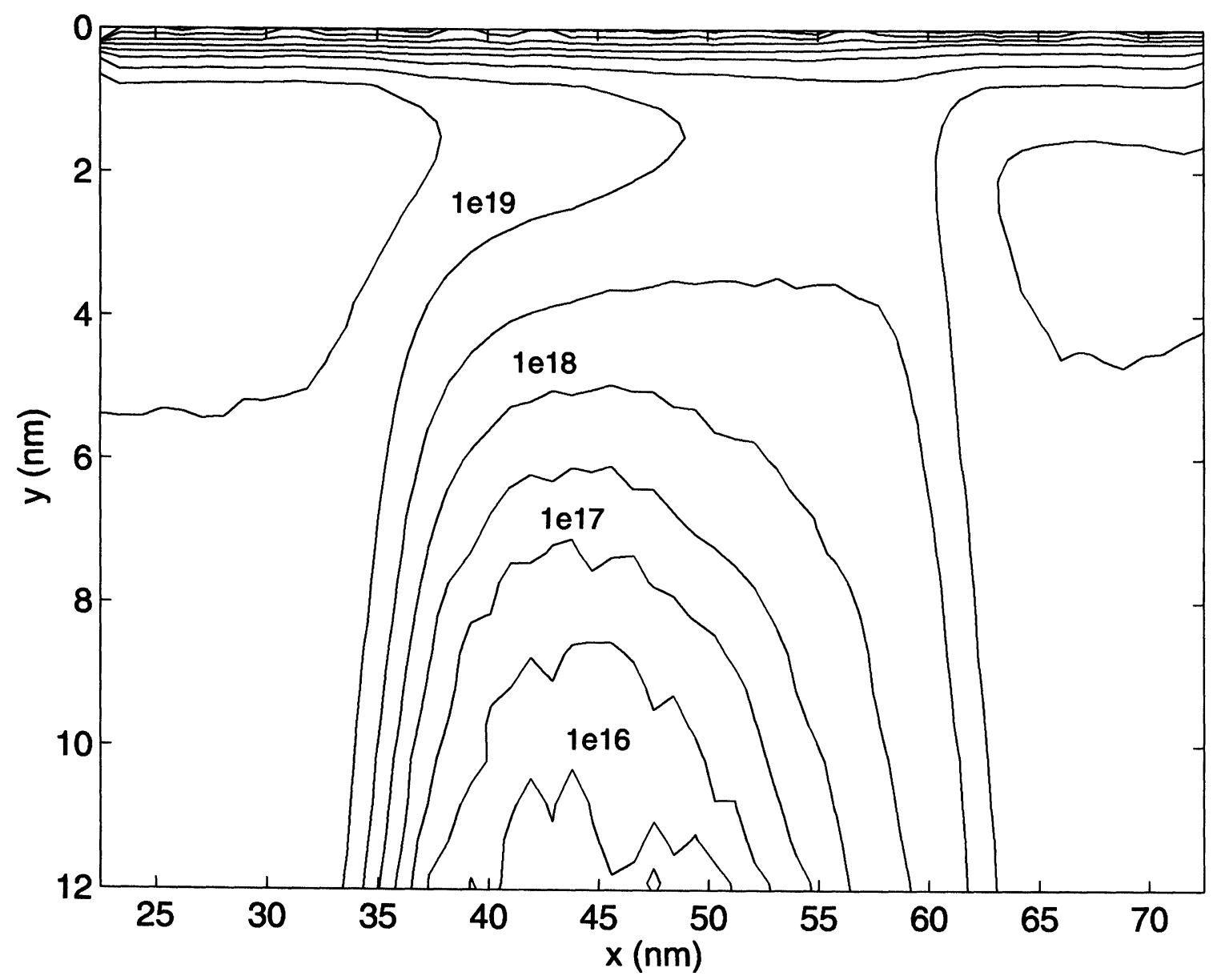

FIGURE 2 (Continued).

applicability range of existing Monte Carlo approaches.

\section{Acknowledgements}

This work was partially supported by the NSF Distributed Center for Advanced Electronics Simulation (DesCArtES), grant NSF ECS 9802730 and by the Semiconductor Research Corporation, contract SRC 99-NJ-726. H. T. would like to thank Prof. T. Miyoshi of Kobe University for his kind support and valuable discussions.

\section{References}

[1] Hess, K., Monte Carlo Device Simulation: Full Band and Beyond (Kluwer Academic Publishers, 1991).

[2] Tsuchiya, H. and Miyoshi, T. (1999). "Quantum Transport Modeling of Ultrasmall Semiconductor Devices", IEICE Trans. Electron., E82-C, 880-888.

[3] Wigner, E. (1932). "On the Quantum Correction for Thermodynamic Equilibrium", Phys. Rev., 40, 749-759.

[4] Tsuchiya, H. and Miyoshi, T. (2000). "Quantum Mechanical Monte Carlo Approach to Electron Transport at Heterointerface", Superlattices and Microstructures, 27(56), $529-532$.

[5] Zhou, J. R. and Ferry, D. K. (1993). "Modeling of Quantum Effects in Ultrasmall HEMT Devices", IEEE Trans. Electron Devices, ED-40, 421-427.

[6] Ancona, M. G. and Iafrate, G. J. (1989). "Quantum Correction to the Equation of State of an Electron Gas in a Semiconductor", Phys. Rev. B, 39, 9536-9540. 

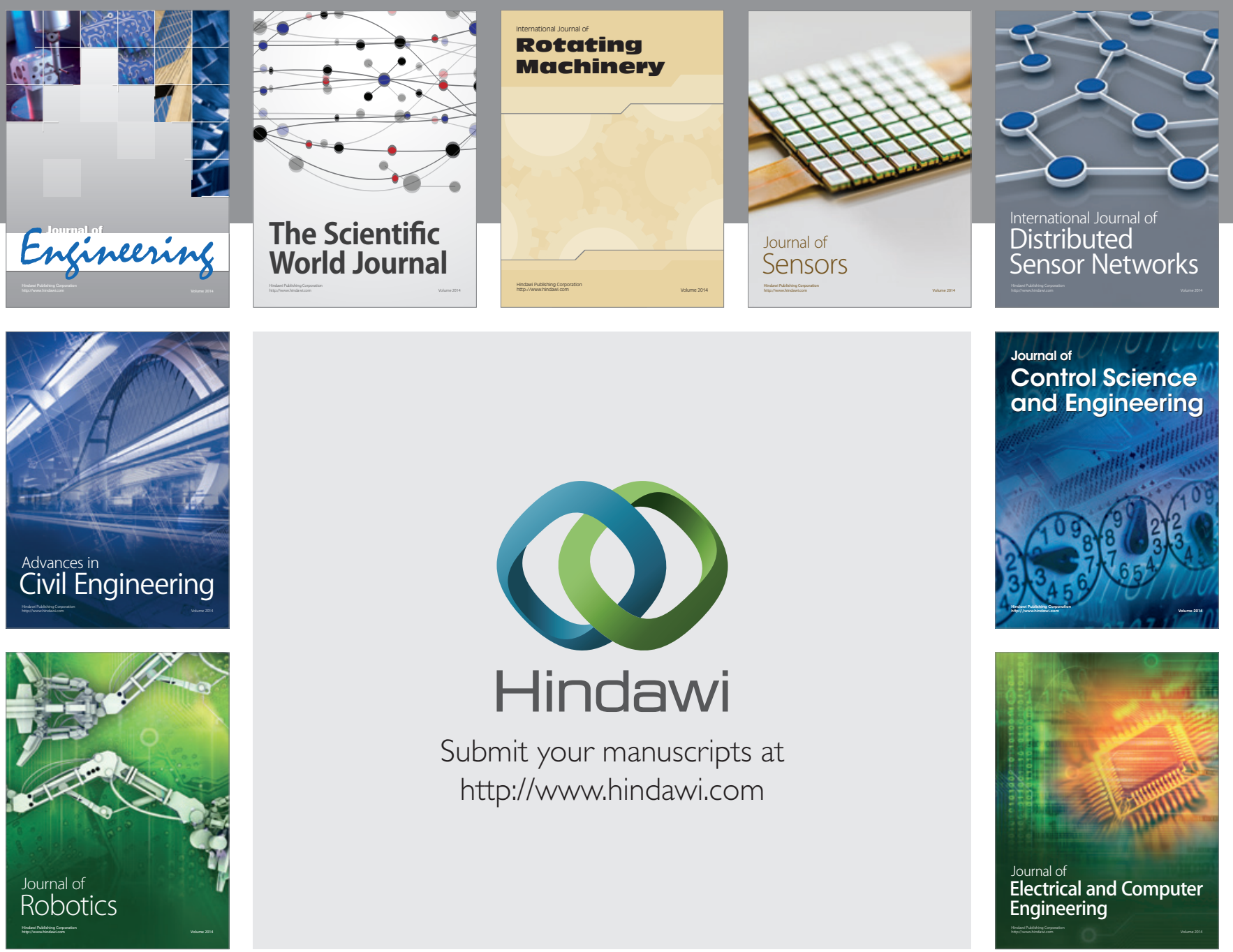

Submit your manuscripts at

http://www.hindawi.com
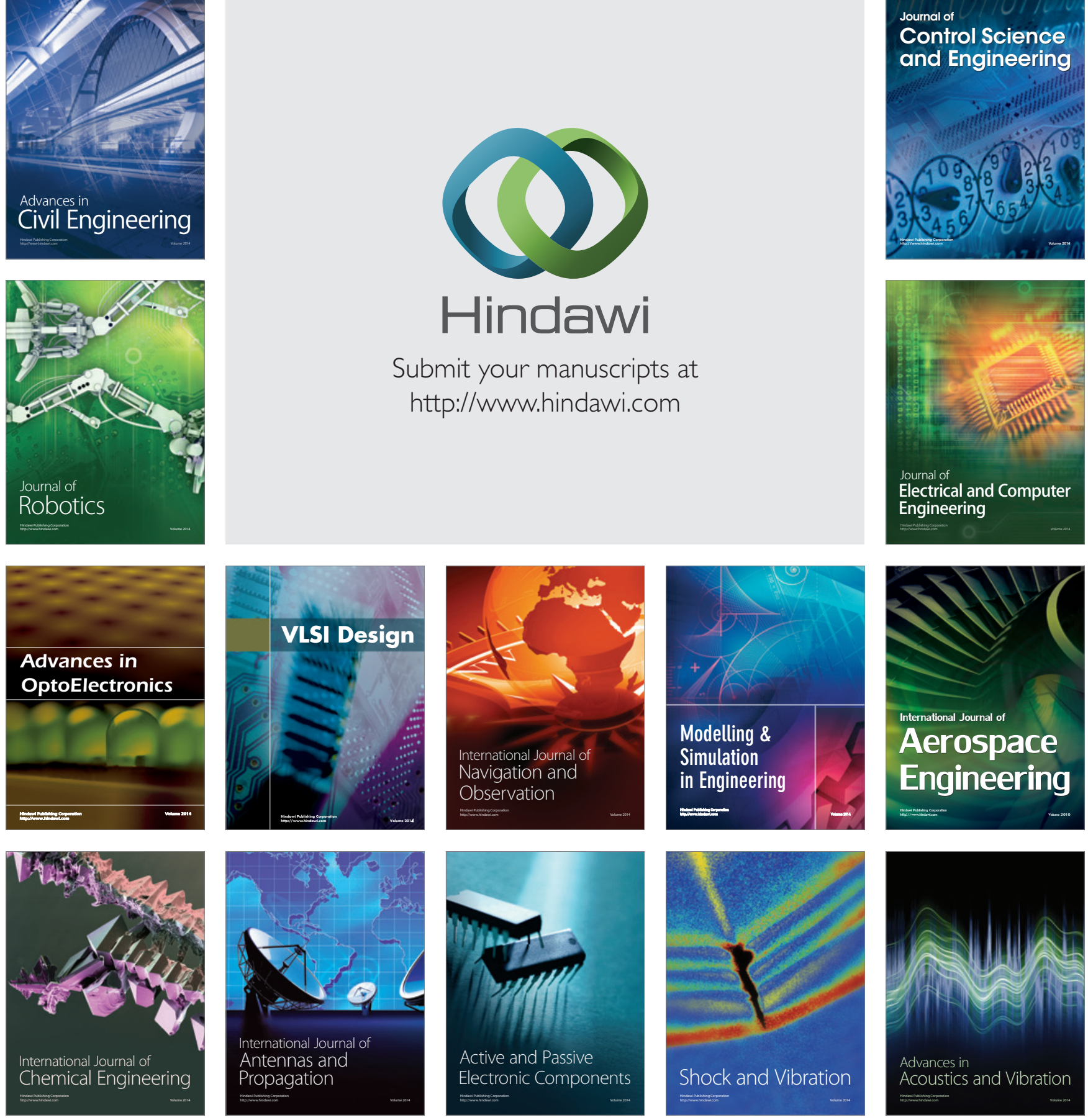